\title{
Individual exposure to particulate air pollution and its relevance to thresholds for health effects: a study of traffic wardens
}

\author{
Monika Watt, David Godden, John Cherrie, Anthony Seaton
}

\begin{abstract}
Objective-To investigate the differences between measurements of personal exposure to particulate air pollution and static area measurements in a group of people working close to traffic and to determine whether such differences might obscure any threshold for health effects in epidemiological studies.

Methods-Personal air sampling was carried out on two groups of eight traffic wardens for four days on two consecutive weeks in November 1994. These measurements were compared with standard environmental static sampling data that were obtained for the same period. A simulation with $\log$ normal distributions of personal exposures was produced, and an arbitrary risk calculated for each exposure, assuming a threshold of $50 \mu \mathrm{g} / \mathrm{m}^{3}$, and an exposure-response curve was calculated.
\end{abstract}

Results-The median concentration for personal samplers in week 1 was $123 \mu \mathrm{g} / \mathrm{m}^{3}$ and $41 \mu \mathrm{g} / \mathrm{m}^{3}$ in week 2. Corresponding area concentrations were $10 \mu \mathrm{g} / \mathrm{m}^{3}$ and $7 \cdot 5$ $\mu \mathrm{g} / \mathrm{m}^{3}$. The differences between the personal and area results were significant, as were the differences for personal sampling between weeks 1 and 2. The simulation showed that the variation in individual exposures around an area sampler obscured the threshold.

Conclusions-Area sampling data may be of limited value in the investigation of the biological effects of exposure to pollution and their use may result in real thresholds being obscured. Personal exposure assessment may be crucial in determination of the health effects attributable to different concentrations of air pollutants.

(Occup Environ Med 1995;52:790-792)

Keywords: personal particulate sampling; environmental pollution; misclassification errors

Population studies have shown temporal associations between outdoor particulate air pollution, respiratory morbidity, ${ }^{1}$ and respiratory and cardiovascular mortality. ${ }^{23}$ These findings have been remarkably consistent across different studies and countries and we have suggested that the mechanism for such health effects may relate to the numbers and biological activity of ultrafine particles deposited in the lung. ${ }^{4}$ Studies to date have not shown any evi- dence of a threshold concentration below which effects are unlikely to occur. This has caused a problem to those who would wish to explain the associations in terms of cause and effect, as it becomes difficult to argue convincingly that serious health effects follow from exposures one or two orders of magnitude lower than those to which industrial workers are exposed, often on a daily basis. If the associations do reflect cause and effect, part of the explanation may lie in the fact that the general population includes people, who for reasons of ill health, are unduly susceptible to adverse effects of pollution. Furthermore, it is likely that an important factor is the actual exposure of individual people to particles rather than the concentrations measured at some distant point for the purposes of air pollution control. For practical reasons, the measurements used in the population studies have been derived from area static sampling, in which particulate air pollution is measured either by the "black smoke" method, or by collecting PM10 (defined as the mass of particles that pass through a size selective orifice with a $50 \%$ collection efficiency at $10 \mu \mathrm{m}$ aerodynamic diameter). In this paper, we describe large differences between area and personal exposures in a group of people working close to traffic, and discuss the influence such differences may have on the possibility of detecting a threshold, should one exist.

\section{Methods}

PARTICLE EXPOSURE OF TRAFFIC WARDENS

Fourteen male and two female traffic wardens, aged between 31 and 58, were enrolled in the study. They were divided into two groups of eight, studied on consecutive weeks during November 1994. Personal air sampling was carried out on four consecutive working days over eight hours from 800 am to $400 \mathrm{pm}$. Battery operated sampling pumps were used, attached to either a cyclone, measuring respirable particulate matter, or a PM10 sampling head. 5 Each traffic warden wore the same sampling equipment for the four days of the study. The sampling flow rate was calibrated with a rotameter each morning before use and remeasured at midday and at 400 $\mathrm{pm}$. The flow rate through the cyclone was set at $1.9 \mathrm{1} / \mathrm{min}$ and through the PM10 head at 2 $1 / \mathrm{min} .{ }^{6}$ Each sampling head was loaded with a preweighed filter paper. After sampling the filters were removed from the heads and reweighed. For each sample a control filter was also weighed before and after sampling. 
The change in weight of these controls was used to correct the mass on the exposed filter for adsorbed moisture. The total pump running time was recorded and the particle concentration calculated in $\mu \mathrm{g} / \mathrm{m}^{3}$.

The results of static sampling of black smoke were obtained from the routine monitoring data of the Environmental Department of Aberdeen City Council. Black smoke was sampled over 24 hours at two sites with an Austin pump and analysed by the reflectance method. ${ }^{7}$ The pumps were positioned about $20 \mathrm{~m}$ from and four metres above roads in the city centre. There are no static site PM10 measurements available for Aberdeen at present.

Results were analysed with Instat Version 2.0 (Graphpad Software, San Diego, CA). As concentrations were non-normally distributed, analysis of variance by ranks (Kruskal-Wallis) was used, and where this was significant, Mann-Whitney tests were used to compare individual groups.

\section{SIMULATION OF EXPOSURES}

We have carried out a simulation of the effects of measurement misclassification that would arise in an exposure-response study where a single measurement of ambient concentration was used as a surrogate for personal exposure. In this we have assumed that the distribution of personal exposures to PM10 in a population is log normal. In practice, some people in a population would have higher exposures than indicated by a fixed location monitoring station and some lower. The exact relation between fixed location monitoring and an exposure distribution for a population is unclear. In this exercise we have chosen to assume that the fixed point concentration would correspond to the geometric mean of the exposure distribution.

The population exposures (E) were then simulated for a given ambient concentration by generating 1000 random numbers from a $\log$ normal distribution with a geometric SD $=2$. This exposure variability is similar to that seen in other studies. ${ }^{8}$ Simulations were carried out for 10 ambient concentrations between 10 and $100 \mu \mathrm{g} / \mathrm{m}^{3}$, giving 10000 individual values for exposure.

For each personal exposure we calculated an arbitrary risk ( $R$ ) that increased linearly with increasing exposure, with a threshold at

Figure 1 Each set of personal data shows the individual measurements and $a$ box and whisker plot.

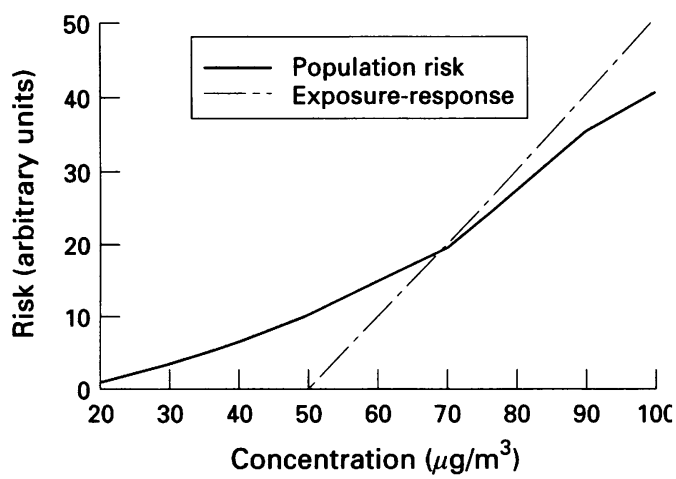

Figure 2 The broken line shows the underlying exposureresponse relation and the solid line the observed relation between concentration and population risk when a single ambient concentration is used as a surrogate for individual exposure.

$50 \mu \mathrm{g} / \mathrm{m}^{3}$ ( $\mathrm{R}=\mathrm{k} . \mathrm{E}-\mathrm{kt}$, where $\mathrm{k}$ and $\mathrm{kt}$ are constants and calculated risks less than zero are set at zero). The apparent risk in the population, for each ambient concentration was then derived from the summation of the individual personal risks. These population risks were then normalised to make them comparable with the exposure-response relation.

\section{Results}

Figure 1 shows the concentrations of airborne particulate matter. For personal samples, the median concentration measured was $123 \mu \mathrm{g} / \mathrm{m}^{3}$ during week 1 and $41 \mu \mathrm{g} / \mathrm{m}^{3}$ during week 2 . Corresponding values for the area samples were $10 \mu \mathrm{g} / \mathrm{m}^{3}$ and $7.5 \mu \mathrm{g} / \mathrm{m}^{3}$ respectively. The overall difference between groups was significant (analysis of variance by ranks, $\mathrm{KW}=$ $24.6, P<0.0001$ ) and subsequent MannWhitney tests confirmed significant differences between personal samples and area samples in both week 1 and week 2 . Median values for personal and area samples were lower during week 2 than week 1 but the differences were significant only for personal samples.

There was no significant difference between the two methods used for measuring particles. Differences in concentrations for smokers and non-smokers, after allowing for the week of study, were also not significant, although only three smokers were studied.

Figure 2 shows the results of the simulation, along with the underlying exposure-reponse relation-that is, $R=k$.E-kt. If everyone in the population were uniformly exposed at the ambient concentration, then the observed exposure-response relation would have been identical to the underlying relation. Because there are always people above and below the mean concentration, some risk is always apparent, even when the ambient concentration-that is, the population geometric mean exposure in our simulation-is below the threshold. The observed risk in figure 2 shows no threshold, even though each individual person's risk is governed by the underlying exposure-response relation. 


\section{Discussion}

This study has shown that concentrations of inhaled particles to which people working in an urban environment were exposed were substantially higher than the routinely measured concentrations that form the basis of published data. ${ }^{9}$ Personal exposures were higher during the first study week compared with the second. Within each week, there was considerable variability, over threefold, between personal exposure measurements.

Our findings are compatible with other studies that have shown that personal sampling gives higher values of particulate exposure than may be expected from area sampling. ${ }^{810}$ In these studies personal exposure measurements were on average two to five times higher than corresponding measurements of area concentration. In our study the personal measurements were about 10 times greater than the static values but allowance must be made for the fact that the personal samples were collected over eight hours whereas the static samples were collected over 24 hours. Even if during the remaining 16 hours of each day the wardens were only exposed to the ambient concentrations measured by the static samplers, their personal exposures would still have been some two to five times greater than the concentrations recorded by the static sampler. Another possible explanation may relate to the differences between black smoke and PM10, by which measure particles on different principles based, respectively, on blackness and size. In general PM10 gives somewhat lower results than black smoke, but there is no constant relation between the two and it is not possible to estimate one from the other. In fact, during the two weeks of the study, particle concentrations measured by static samplers in Edinburgh for PM10 were 25 and $17 \mu \mathrm{g} / \mathrm{m}^{3}$ and for black smoke were 9.4 and $2.5 \mu \mathrm{g} / \mathrm{m}^{3}$, which showed that sometimes PM10 may give the higher result.

The differences between the first and second weeks of our study were also surprising, in that exposures were clearly higher in the first, during which there was almost constant rain. The fact that this did not reduce particle concentrations suggests that they were predominantly in the very fine size range, as might be expected close to traffic sources.

Taking account of these possible explanations, another likely factor responsible for the differences between static and personal samples and for the variability between subjects is the proximity of individual wardens to the main source of pollution, motor vehicle emissions. The black smoke monitoring stations in Aberdeen are located about $20 \mathrm{~m}$ from roads in the city centre, whereas the wardens spent much of their time at the kerb, within 1-2 m of moving vehicles. It is perhaps not surprising that people working in close proximity to traffic are exposed to higher concentrations of particles than is a static sampler several meters away. The magnitude of the difference and the variability between subjects was greater than expected.
There is an important implication of these findings if they represent the general exposures of people working or living close to traffic. The epidemiological findings that associate concentrations of pollutant to risk of ill health may give a very poor indication of exposure of the population, and will depend critically on the distribution of individual exposures around the recorded value. The consequence of assigning an ambient concentration to individual people is analogous to non-differential misclassification, which is well known to bias risk estimates towards the null value - that is, to obscure exposure-response relations. ${ }^{11}$ It is likely that, even at a population level, there is a concentration of particles insufficient to cause detectable ill health. If such a concentration does indeed exist, it may not be possible to show it in a study with static measurements of ambient air pollutant as a surrogate for individual exposures and this is shown by our simulation in which, given the wide distribution of exposures likely in an urban population, a real threshold may well be obscured.

If, as seems likely, the observed temporal associations between the concentration of particulate matter and morbidity or mortality are causal, then differences in exposure between individual people will be important. In particular those at risk, such as people with asthma and other chronic respiratory and cardiovascular diseases, may be more likely to be affected by particulate pollution if they live or work close to busy roads or other sources of fine particles. It is in our view desirable for future studies of the health effects of air pollution to take account of the problem of non-differential misclassification of exposure and to include estimates of the personal exposures of people or groups in the investigation.

We are grateful to Mrs Aileen Osborne and Jill Seaton for their technical assistance during this study; Roland Douglas, Steve Parkinson, John Gannon, and Eric Hampton for the area particle monitoring data; and Dr Robert Aitken of the Institute of Occupational Medicine, Edinburgh, for the loan of sampling equipment.

1 Pope CA, Kanner RE. Acute effects of PM10 pollution on pulmonary function of smokers with mild to moderate chronic obstructive pulmonary disease. Am Rev Respir chronic obstructive pulm

2 Schwartz J. Air pollution and daily mortality: a review and meta-analysis. Environ Res 1994;64:36-52.

3 Schwartz J. What are people dying of on high pollution days? Environ Res 1994;64:26-35.

4 Seaton A, MacNee W, Donaldson K, Godden D Particulate air pollution and acute health effects: hypothesis. Lancet 1995;345:176-8

5 Health and Safety Executive. General methods for the gravimetric determination of respirable and total inhalable dust. metric determination of respirable and

6 Aitken RJ, Vincent JH, Mark D, Application of porous foams as size selectors for biologically relevant samplers. foams as size selectors for biologically

7 British Standard. Determination of concentrations of suspended matter. London: British Standards Institutation pended matter. London:

8 Lioy PJ, Waldman JM, Buckley T, Butler J, Pietarinen C. The personal, indoor and outdoor concentrations of PM10 measured in an industrial community during the winter. Atmospheric Environment 1990;24:57-66.

9 Quality of Urban Air Review Group. Urban air quality in the United Kingdom. London; Department of the Environment, 1993.

10 Spengler JD, Ferris BG. Harvard air pollution health study in six cities in the USA. Tokai' $f$ Exp Clin Med 1985; 10:263-86.

11 Armstrong B. Effects of measurement errors on estimates of exposure-response relationships. Recent Results Cancer Res 1990;120:50-63. 